\title{
Efficacy, Safety, and Tolerability of Switching from Oral Cholinesterase Inhibitors to Rivastigmine Transdermal Patch with 1-Step Titration in Patients with Mild to Moderate Alzheimer's Disease: A 24-Week, Open-Label, Multicenter Study in Japan
}

\author{
Kengo Ueda ${ }^{a}$ Sadao Katayama ${ }^{\text {b }}$ Tetsuaki Arai ${ }^{c}$ Nobuo Furuta ${ }^{d}$ \\ Shinichiro Ikebe ${ }^{e}$ Yoshinori Ishida $^{f}$ Kiyoshi Kanayag Shinji Oumah \\ Hirofumi Sakurai ${ }^{i}$ Masato Sugitani ${ }^{j}$ Makio Takahashik ${ }^{k}$ Toshihisa Tanakal \\ Norifumi Tsuno ${ }^{m}$ Yosuke Wakutani ${ }^{n}$ Ankita Shekhawat ${ }^{\circ}$ Ayan Das Gupta ${ }^{\circ}$ \\ Kazuki Kiyose $^{a} \quad$ Kazuhiro Toriyama $^{\text {a }}$ Yu Nakamuram \\ ${ }^{a}$ Novartis Pharma K.K., Tokyo, Japan; ${ }^{b}$ Katayama Medical Clinic, Okayama, Japan; ${ }^{\text {CFaculty }}$ \\ of Medicine, University of Tsukuba, Ibaraki, Japan; dYokufukai Hospital, Tokyo, Japan; \\ e Ikebe Clinic, Shizuoka, Japan; ${ }^{\mathrm{T}}$ Takeda General Hospital, Fukushima, Japan; ${ }^{9}$ Hachioji \\ Medical Center, Geriatric Medicine, Tokyo Medical University, Tokyo, Japan; hepartment \\ of Neurology, Fukuoka University, Fukuoka, Japan; 'Department of Geriatric Medicine, \\ Tokyo Medical University, Tokyo, Japan; jSagamihara Kyodo Hospital, Kanagawa, Japan; \\ kDepartment of Neurology, Osaka Red Cross Hospital, Osaka, Japan; 'Department of \\ Psychiatry, Osaka University Graduate School of Medicine, Osaka, Japan; ${ }^{m}$ Department of \\ Neuropsychiatry, Kagawa University School of Medicine, Kagawa, Japan; ${ }^{n}$ Department of \\ Neurology, Kurashiki Heisei Hospital, Okayama, Japan; ${ }^{\circ}$ Novartis Healthcare Pvt. Ltd., Data \\ Sciences, SSP, PLS, Hyderabad, India
}

Keywords

Rivastigmine transdermal patch $\cdot$ Alzheimer's disease $\cdot$ Cholinesterase inhibitors $\cdot$ Switching

\section{Abstract}

Background: Few studies have investigated treatment options for patients with Alzheimer's disease (AD) showing a poor response to oral cholinesterase inhibitors (ChEls) in Japan. Objective: To investigate the efficacy and safety of switching from oral ChEls to rivastigmine transdermal patch in patients with AD. Methods: In this multicenter, open-label, phase IV study in outpatient clinics in Japan, patients with mild-moderate AD who had a poor response to or experienced difficulty in continuing donepezil or galantamine were switched to rivastigmine transdermal patch $\left(5 \mathrm{~cm}^{2}\right.$; loaded dose $9 \mathrm{mg}$, delivery rate $4.6 \mathrm{mg} / 24 \mathrm{~h}$ ) with a 1-step titration in week $4\left(10 \mathrm{~cm}^{2}\right.$; loaded dose $18 \mathrm{mg}$, delivery rate $\left.9.5 \mathrm{mg} / 24 \mathrm{~h}\right)$, which was contin- 
Ueda et al.: Switching to Rivastigmine Transdermal Patch in Mild to Moderate Alzheimer's Disease

ued for 4 weeks in the titration period and 16 weeks in a maintenance period. The primary endpoint was the change in Mini-Mental State Examination (MMSE) total score from baseline to week 24. Results: A total of 118 patients were enrolled and switched to rivastigmine, of which 102 completed the 24-week study. The MMSE total score was essentially unchanged during the study, with a least-square mean change (SD) of $-0.35(2.64)$ at week $24(p=0.1750)$. Exploratory analysis with a mixed-effect model comparing changes in MMSE between the pre- and post-switch periods suggested that switching to rivastigmine prevented a worsening of MMSE. Application site skin reactions/irritations occurred in $30.5 \%$ of patients overall, in $22.0 \%$ in the 8 -week titration period, and in $10.2 \%$ in the 16 -week maintenance period. Conclusion: Within-class switching from an oral $\mathrm{ChEl}$ to rivastigmine transdermal patch might be an efficacious and tolerable option for AD patients showing a poor or limited response to a prior oral ChEl.

\section{Introduction}

The use of cholinesterase inhibitors (ChEIs) to treat Alzheimer's disease (AD) has increased steadily over the last two decades since the approval of donepezil in 1999 and galantamine and rivastigmine in 2011 in Japan [1]. However, it is estimated that a large proportion of patients experience a lack or loss of efficacy to the ChEI. Open-label studies in Europe and North America indicated that about $50 \%$ of patients who had experienced a lack or loss of efficacy to donepezil responded to treatment with rivastigmine [2]. Accordingly, back in 2003, a panel of experts proposed switching ChEIs in patients with a limited response to their therapy [2], an approach supported by clinical studies [3] and a recent review of the literature [4]. However, while switching ChEIs is recommended in treatment guidelines for AD, it is not routinely performed [5].

Switching to rivastigmine transdermal patch from donepezil, galantamine, or oral rivastigmine was shown to be an effective option in prior studies in Europe/North America [6-8]. However, except for a study in South Korea [9], the efficacy and safety of this approach has not been well examined in $\mathrm{AD}$ patients in Asia including Japan, where the ethnic backgrounds and medical settings differ from those of Western countries. Therefore, we performed a phase IV study to investigate the efficacy and safety of switching to rivastigmine transdermal patch in patients who were on a stable dose of donepezil or galantamine for $>4$ weeks but did not receive a significant clinical benefit from those oral ChEIs. Rivastigmine transdermal patch was approved in Japan following the results of a 24-week clinical trial that examined the efficacy and safety of two dose levels ( 9 and $18 \mathrm{mg}$ ) of rivastigmine [10]. This study led to the approval of a three-step titration algorithm, starting from $4.5 \mathrm{mg}$, then increasing stepwise to $9,13.5$, and finally $18 \mathrm{mg}$. This approach takes a minimum of 12 weeks to reach the effective dose (18 mg loaded dose; $9.5 \mathrm{mg} / 24 \mathrm{~h}$ delivery rate). Therefore, a recent study investigated the safety and efficacy of an abbreviated, one-step titration algorithm in which dosing starts at $9 \mathrm{mg}$ and is increased to the effective dose 4 weeks after starting treatment [11]. This one-step titration algorithm was approved in Japan in 2015 and was used in the present study to confirm that the effective dose could be reached quickly in an outpatient setting.

We hypothesized that treatment with rivastigmine transdermal patch for 24 weeks after switching from an oral $\mathrm{ChEI}$ will attenuate the decline in cognitive function (determined using the Mini-Mental State Examination; MMSE) in patients with AD who experienced a decline in cognitive function (assessed by MMSE) and/or aggravation of activities of daily living (ADL) or behavioral and psychological symptoms of dementia (BPSD). 
Ueda et al.: Switching to Rivastigmine Transdermal Patch in Mild to Moderate Alzheimer's Disease

\section{Materials and Methods}

\section{Patients}

Key eligibility criteria are summarized in online supplementary Table S1 (for all online suppl. material, see www.karger.com/doi/10.1159/000501364). Briefly, patients were eligible if they were aged 50-85 years, had a diagnosis of dementia of Alzheimer's type (according to the Diagnostic and Statistical Manual of Mental Disorders, 4th edition) and probable AD (according to National Institute of Neurological and Communicative Disorders and Stroke - Alzheimer's Disease and Related Disorders Association), and their MMSE score was 10-23 at screening and baseline. In addition, patients needed to be on oral donepezil (5 mg) or oral galantamine (16-24 $\mathrm{mg}$ ) at stable doses for $\geq 4$ weeks before the baseline visit, with no or a poor response to these treatments, or experienced difficulty using oral drugs for the reasons given in online supplementary Table S1. Patients on memantine were also eligible, providing they had used a stable dose for $\geq 4$ weeks before baseline that was to be continued throughout the study.

\section{Study Design}

This open-label, single-group study was performed with dementia specialists at 20 outpatient hospitals and clinics in Japan. It comprised a screening visit, an 8-week titration period, and a 16-week maintenance period (online suppl. Fig. S1). Patient eligibility, demographics, medical history, treatment history, brain MRI scan (if the patient's most recent scan was taken $>3$ years earlier), and MMSE were recorded at the screening visit 2-4 weeks before the planned start of rivastigmine. On day 1 in the titration period, the patient's prior oral ChEI was discontinued and replaced with a $5 \mathrm{~cm}^{2}$ rivastigmine transdermal patch (loaded dose 9 $\mathrm{mg}$, delivery rate $4.6 \mathrm{mg} / 24 \mathrm{~h}$ ), which was to be applied every $24 \mathrm{~h}$. On day 29 (week 5), patients were switched to the $10 \mathrm{~cm}^{2}$ rivastigmine transdermal patch (loaded dose $18 \mathrm{mg}$, delivery rate $9.5 \mathrm{mg} / 24 \mathrm{~h}$ ) in a one-step titration scheme for 4 weeks (titration period). Dose reductions, temporary interruption, or treatment discontinuation were also permitted if deemed necessary by the investigator. The dose used at the end of the titration period was to be continued for 16 weeks (maintenance period) through to study week 24 .

The rivastigmine transdermal patch was applied to patients' back, upper arm, or chest. Topical treatments, including steroids, antihistamines, and heparin-like substances could be administered if necessary. Heparin-like substances were the only products used by patients as moisturizers in this study.

\section{Endpoints}

The primary endpoint was the change in MMSE total score from baseline to Week 24. Secondary endpoints included the following: the change in MMSE total score from baseline to week 8; and changes in Neuropsychiatric Inventory-10 (NPI-10) from baseline to weeks 8 and 24; Quality of Life-Alzheimer's Disease Scale (QOL-AD) scores at week 24; changes in the Japanese version of the Clinical Global Impression of Change (J-CGIC) and modified Crichton Scale score from baseline to weeks 4, 8, 16, and 24; and caregiver-assessed usability of rivastigmine transdermal patch. As an exploratory endpoint, we also compared the change in MMSE between baseline and week 24 with the change observed over 24 weeks before study enrolment in patients with available data. Safety was assessed throughout the study in terms of adverse events (AEs), serious AEs, AEs of special interest (skin-related and gastrointestinal [GI]-related AEs), laboratory/biochemistry, and vital signs. Additional information on study assessments is given in the online supplementary Methods.

Statistical analyses are described in the supplementary Methods. 
Ueda et al.: Switching to Rivastigmine Transdermal Patch in Mild to Moderate Alzheimer's Disease

Fig. 1. Patient disposition.

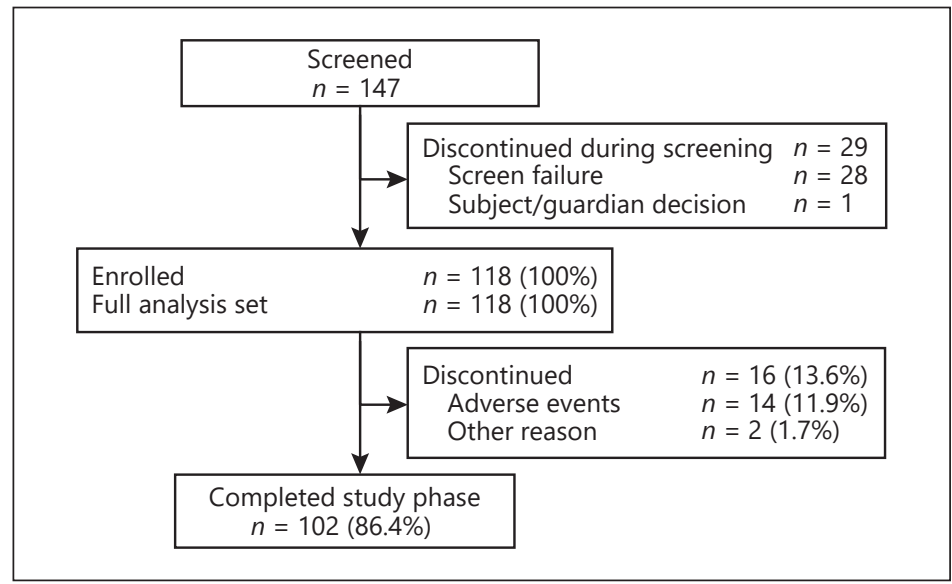

Table 1. Reasons for enrolment into the study

\begin{tabular}{|c|c|c|}
\hline \multicolumn{2}{|c|}{$\begin{array}{l}\text { Reasons for enrolment } \\
\text { (multiple reasons permitted) }\end{array}$} & $n(\%)$ \\
\hline A & Lack or loss of efficacy & $112(94.9)$ \\
\hline$A-1$ & $\begin{array}{l}\geq 2 \text {-point decline of MMSE within the } \\
\text { first } 3 \text { months with previous treatment }\end{array}$ & $2(1.7)$ \\
\hline A-2 & $\begin{array}{l}\geq 2 \text {-point decline of MMSE within } \\
6 \text { months with previous treatment }\end{array}$ & $46(39.0)$ \\
\hline A-3 & Aggravation of ADL/BPSD & $86(72.9)$ \\
\hline$A-1$ and $A-3$ & & $2(1.7)$ \\
\hline A-2 and $A-3$ & & $20(16.9)$ \\
\hline B & Difficulty in continuing treatment & $10(8.5)$ \\
\hline B-1 & Poor compliance & $8(6.8)$ \\
\hline B-2 & Non-gastrointestinal AEs & $2(1.7)$ \\
\hline$A$ and $B$ & & $4(3.4)$ \\
\hline A-2 and B-1 & & $1(0.8)$ \\
\hline$A-3$ and $B-1$ & & $3(2.5)$ \\
\hline
\end{tabular}

MMSE, Mini-Mental State Examination; ADL, activities of daily living; BPSD, behavioral and psychological symptoms of dementia; $\mathrm{AE}$, adverse event.

\section{Results}

\section{Patients}

The study was conducted between May 9, 2016, and May 7, 2018. Of 147 patients screened, 118 were enrolled and comprised the full analysis and safety analysis sets (Fig. 1). Of these, 102 completed the study period. Fourteen patients discontinued due to AEs. Most of the patients were enrolled due to lack or loss of efficacy of the prior oral ChEI $(n=112$; 94.9\%), while 10 patients (8.5\%) experienced difficulty with their prior therapy (Table 1).

Patient characteristics are presented in Table 2. The mean age was 76.4 years, $60.2 \%$ of patients were female, and nearly all (94.9\%) were living with a caregiver or other individual. The mean time since the symptoms were first observed by a caregiver was 4.3 years, and the mean time since diagnosis was 2.5 years. MMSE total score at baseline was 17.3 and ranged from 10 to 23 . Prior therapy was donepezil in $61.0 \%$ and galantamine in $39.0 \%$. One-third (35.6\%) of 
Dementia

Cognitive Disorders

Table 2. Patient characteristics $(n=118)$

\begin{tabular}{l|l}
\hline Dement Geriatr Cogn Disord Extra 2019;9:302-318 \\
\hline DOI: 10.1159/000501364 & $\begin{array}{l}\text { @ 2019 The Author(s). Published by S. Karger AG, Basel } \\
\text { www.karger.com/dee }\end{array}$ \\
\hline
\end{tabular}

Ueda et al.: Switching to Rivastigmine Transdermal Patch in Mild to Moderate Alzheimer's Disease

\begin{tabular}{|c|c|}
\hline \multicolumn{2}{|l|}{ Age, years } \\
\hline Mean (SD) & $76.4(6.43)$ \\
\hline Range & $54-85$ \\
\hline \multicolumn{2}{|l|}{ Sex, $n(\%)$} \\
\hline Male & $47(39.8)$ \\
\hline Female & $71(60.2)$ \\
\hline Body weight, mean (SD), kg & $54.1(10.5)$ \\
\hline \multicolumn{2}{|l|}{ Weight category } \\
\hline$<40 \mathrm{~kg}$ & $13(11.0)$ \\
\hline 40 to $<50 \mathrm{~kg}$ & $30(25.4)$ \\
\hline 50 to $<60 \mathrm{~kg}$ & $36(30.5)$ \\
\hline$\geq 60 \mathrm{~kg}$ & $39(33.1)$ \\
\hline \multicolumn{2}{|l|}{ Patient's current living status } \\
\hline Living alone & $5(4.2)$ \\
\hline Living with a caregiver or other individual & $112(94.9)$ \\
\hline Nursing home or long-term institution & $1(0.8)$ \\
\hline \multicolumn{2}{|l|}{ Education history, $n(\%)$} \\
\hline 5-9 years & $4(3.4)$ \\
\hline $9-12$ years & $85(72.0)$ \\
\hline $13-16$ years & $26(22.0)$ \\
\hline$>16$ years & $3(2.5)$ \\
\hline \multicolumn{2}{|l|}{ Time since first symptoms of AD, years ${ }^{a}$} \\
\hline Mean (SD) & $4.3(2.90) / 2.5(2.07)$ \\
\hline Range & $0.5-12.5 / 0.1-10.3$ \\
\hline \multicolumn{2}{|l|}{ Category } \\
\hline$<1$ & $6(5.1) / 30(25.4)$ \\
\hline $1-2$ & $20(16.9) / 25(21.2)$ \\
\hline $2-3$ & $27(22.9) / 30(25.4)$ \\
\hline $3-4$ & $15(12.7) / 11(9.3)$ \\
\hline $4-5$ & $14(11.9) / 10(8.5)$ \\
\hline$>5$ & $26(22.0) / 11(9.3)$ \\
\hline$>10$ & $10(8.5) / 1(0.8)$ \\
\hline \multicolumn{2}{|l|}{ MMSE total score at baseline } \\
\hline Mean (SD) & $17.3(3.80)$ \\
\hline Range & $10-23$ \\
\hline \multicolumn{2}{|l|}{ Prior therapy, $n(\%)$} \\
\hline Donepezil & $72(61.0)$ \\
\hline Galantamine & $46(39.0)$ \\
\hline \multicolumn{2}{|l|}{ Concomitant use of memantine, $n(\%)$} \\
\hline Yes & $42(35.6)$ \\
\hline No & $76(64.4)$ \\
\hline
\end{tabular}

patients were on concomitant memantine during the study. Patients did not require medication for the management of neuropsychiatric symptoms except for three who used risperidone.

The overall mean compliance rate was $97.6 \%$ and was between 75 and $100 \%$ in 113 $(95.8 \%)$ patients. The compliance rate was $25-50 \%$ in 3 patients $(2.5 \%)$ and $50-75 \%$ in 2 patients $(1.7 \%)$.

\section{MMSE Total Scores}

As shown in Figure 2, the MMSE total score was fairly stable throughout the study, with a least-squares mean change of 0.29 at week 8 ( $p=0.1512 \mathrm{vs}$. baseline) and -0.35 at week 24 


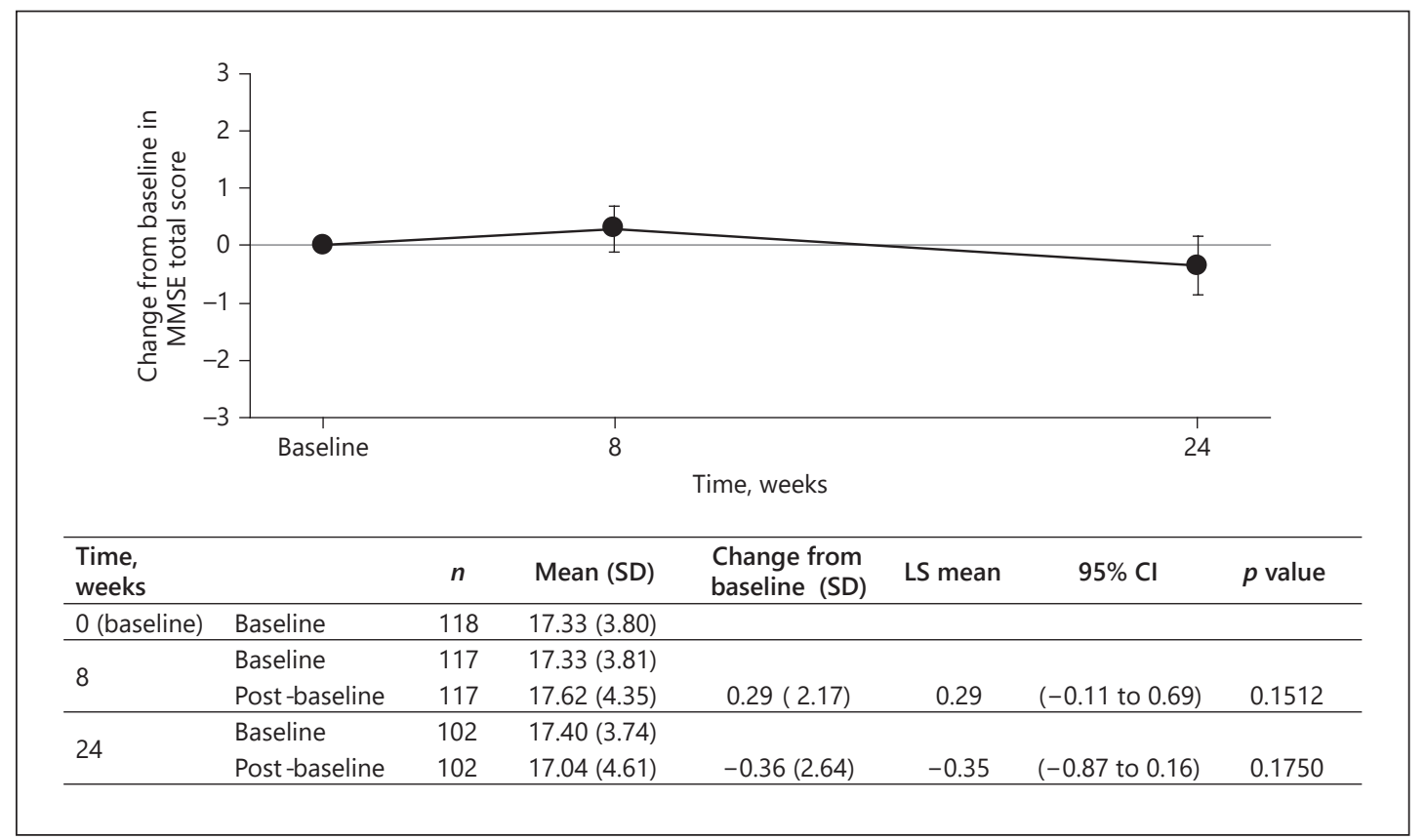

Fig. 2. Change in MMSE total score from baseline. The primary endpoint was the change in the MMSE total score from baseline to week 24 in the full analysis set. The analysis was performed using a mixed-effects model for repeated measures, with visit as a fixed effect, baseline MMSE total score as a covariate, and subject as a random effect. An unstructured covariance matrix was used to construct an intrasubject error model. Least-squared means of the changes at week 8 and week 24 are shown along with the $95 \%$ two-sided confidence interval and $p$ value.

( $p=0.1750$ vs. baseline). The overall MMSE responder rate was 37.3\% (95\% confidence interval [CI] 28.6-46.7\%; $n=44)$, which included 3 patients with no change $(2.5 \%$; $95 \%$ CI $0.5-7.3 \%$ ) and 41 patients with an improvement (34.8\%; 95\% CI 26.2-44.1\%) in MMSE at week 24 relative to baseline.

In an exploratory analysis, it was apparent that there was a marked reduction in MMSE total score over 24 weeks before study enrolment and that MMSE remained stable during the 24-week study period (Fig. 3 ), with changes of $-1.80(95 \%$ CI $-2.37,-1.23 ; n=84)$ and -0.08 $(95 \% \mathrm{CI}-0.53,0.37 ; n=118)$, respectively. This analysis suggests that patients experienced marked worsening during their prior ChEI therapy, and that ongoing decline was prevented by switching to rivastigmine.

To further investigate the effects of switching to rivastigmine transdermal patch, we also compared the changes in MMSE between subgroups of patients divided by baseline MMSE category, reason for study inclusion, age, use of skin moisturizer at baseline, and compliance, which were planned prior to the study (Fig. 4 and online suppl. Fig. S2). As indicated, there were some differences among subgroups, with tendencies towards greater attenuation in decline of MMSE in patients with higher baseline MMSE total scores and in patients enrolled due to a $\geq 2$-point decline of MMSE within 6 months with previous treatment (i.e., reason A-2) (Fig. 4). However, it must be acknowledged that some of these subgroups included small numbers of patients and thus showed large 95\% CIs.

\section{Secondary Efficacy Endpoints}

Figure 5 and online supplementary Table S2-S4 show the results of the secondary endpoints NPI-10, QOL-AD, modified Crichton Scale, and J-CGIC. As shown in Figure 5a, there 
Fig. 3. Change in MMSE total score in 6 months prior to baseline and in 6 months after switching. Mixed-effects model for repeated measures of changes in MMSE total score in the pre-baseline and post-baseline periods. Changes in MMSE total scores before and after switch were analyzed using a mixed-effects model, which included the pre-switch period and post-switch period as separate fixed effects, and an intercept estimated for each patient. The slopes of the lines before and after switch were estimated using the model. Shown here are the estimates with 95\% two-sided confidence interval.

\begin{tabular}{l|l}
\hline Dement Geriatr Cogn Disord Extra 2019;9:302-318 \\
\hline DOI: 10.1159/000501364 & $\begin{array}{l}\text { (c) 2019 The Author(s). Published by S. Karger AG, Basel } \\
\text { www.karger.com/dee }\end{array}$ \\
\hline
\end{tabular}

Ueda et al.: Switching to Rivastigmine Transdermal Patch in Mild to Moderate Alzheimer's Disease

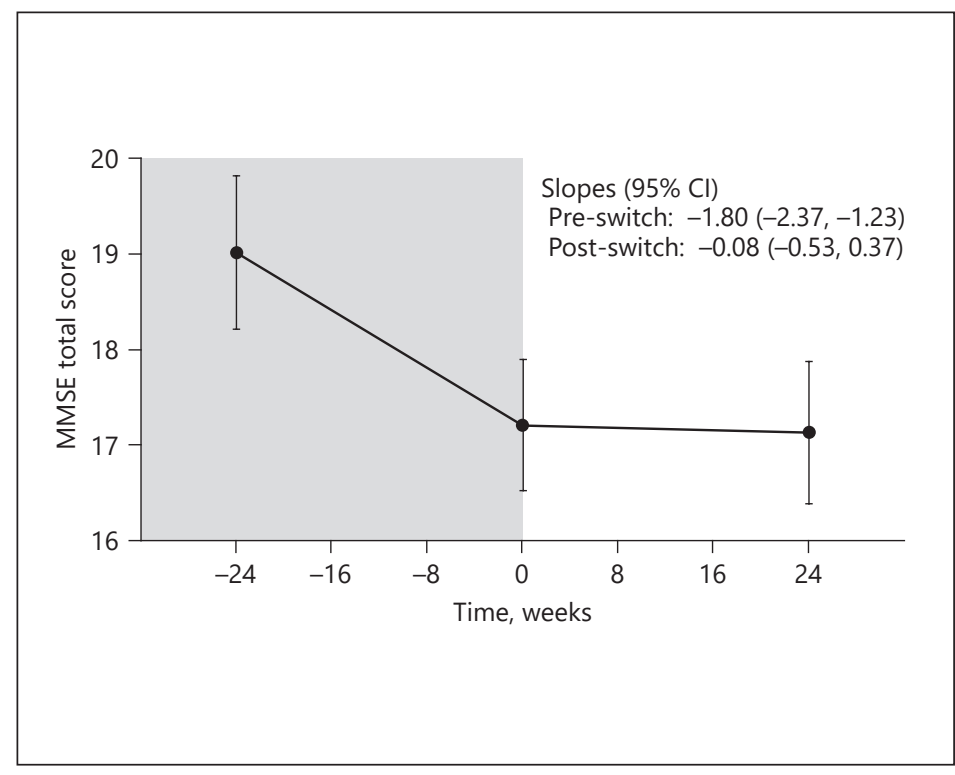

Fig. 4. Subgroup analysis with baseline patient characteristics. Changes in MMSE total score from baseline (week 0) to weeks 8 and 24 according to baseline MMSE category (a) and reason for inclusion (b) (Table 1). Online supplementary Figure S2 shows changes in MMSE scores in patients divided according to age, use of skin moisturizer at baseline/during the study, and compliance. For all analyses, the mixed-effects model with repeated measures was used. Least-squared means of the changes are shown along with the $95 \%$ two-sided confidence interval.

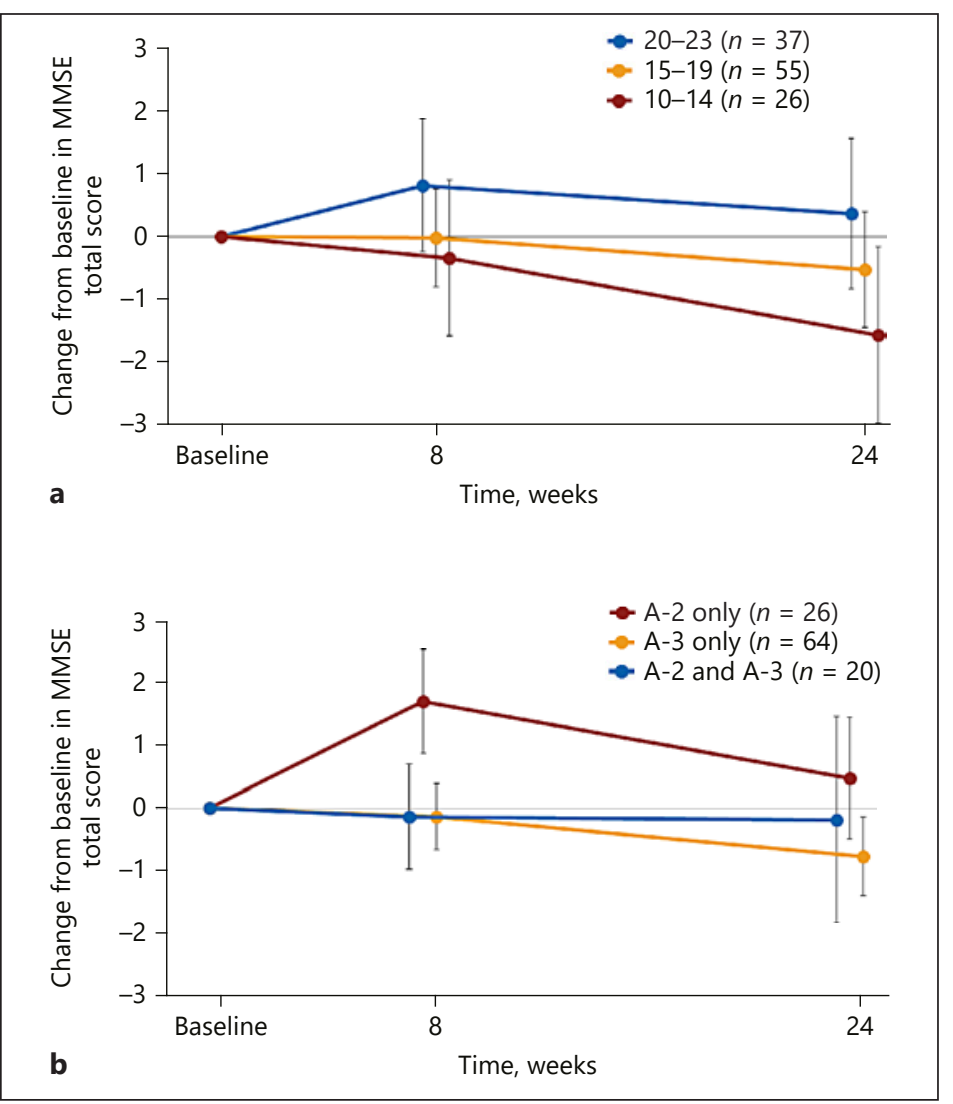

was an improvement in the NPI-10 total score from baseline through to week 8, which returned to the baseline level at week 24 ; the mean $(95 \% \mathrm{CI})$ scores at each time point were 11.4 (9.4-13.5), 9.7 (7.6-11.8), and 10.1 (7.7-12.5), respectively. There was a tendency toward improvements (i.e., reductions in scores) for several domains, notably apathy/indif- 


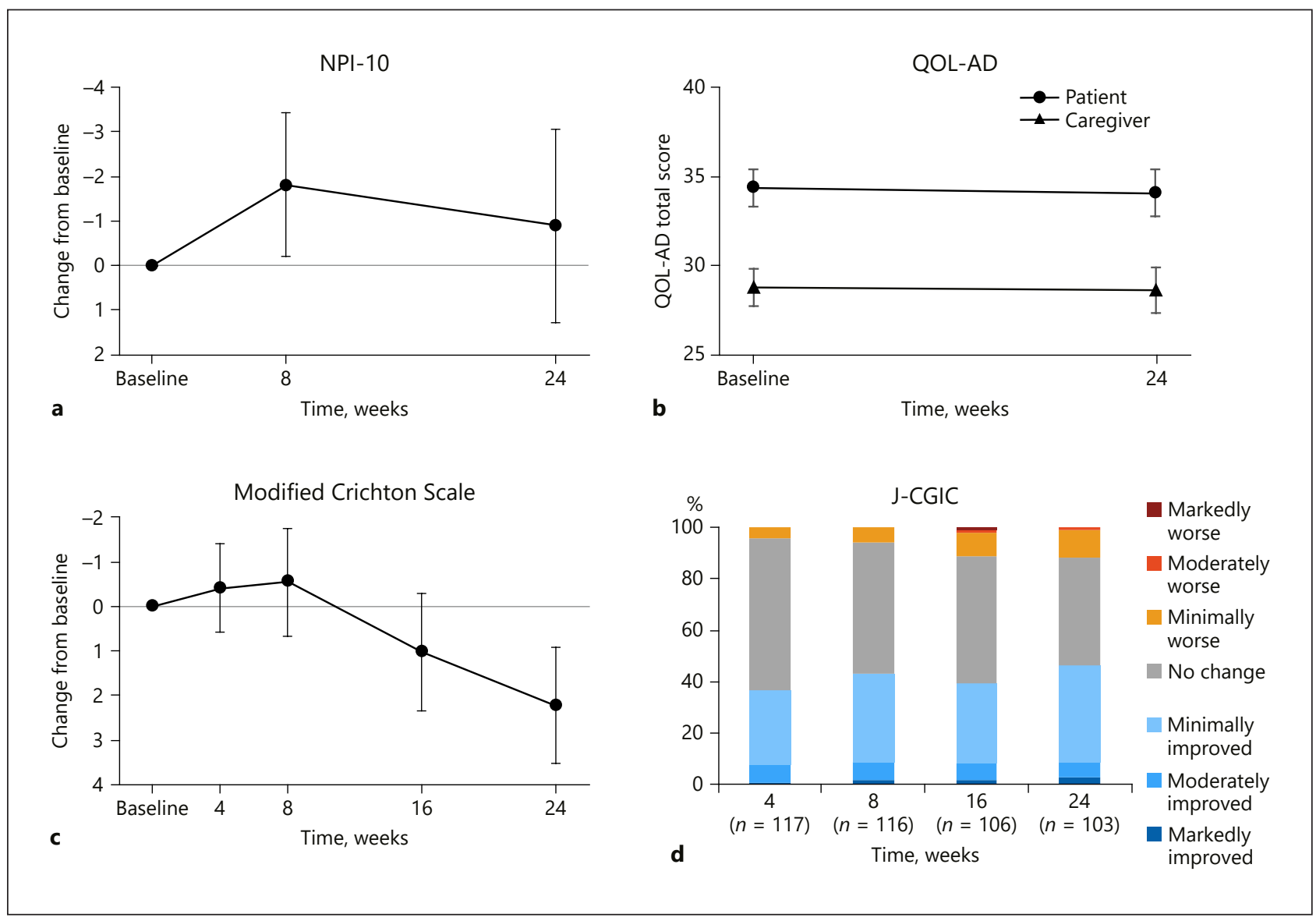

Fig. 5. Results of secondary endpoints. a Changes in Neuropsychiatric Inventory 10 (NPI-10) from week 0 (baseline) to weeks 8 and 24. b Changes in Quality of Life-Alzheimer's Disease (QOL-AD) scores from week 0 (baseline) to week 24. c Changes in modified Crichton Scale scores from week 0 (baseline) to weeks 4, 8, 16, and 24. d Japanese Clinical Global Impression of Change (J-CGIC) at weeks 4, 8, 16, and 24. Values are expressed as least-squared mean and $95 \%$ confidence intervals (a-c) or as percent of patients (d). Online supplementary Tables S2-S4 report additional data for each secondary endpoint.

ference and anxiety, although none of those improvements in subdomains reached statistical significance (online suppl. Table S3).

The caregiver-assessed and patient-assessed QOL-AD total scores were fairly stable during the study, with mean $(95 \% \mathrm{CI})$ changes of $-0.16(-1.21,0.90)$ and $-0.34(-1.39$, 0.70 ), respectively, from baseline to week 24 (Fig. 5b, online suppl. Table S2). Although the changes are similar, caregivers tended to rate the score lower (i.e., worse) than did the patients.

The total modified Crichton Scale score was initially maintained from baseline through to week 8, followed by increases in scores (i.e., worsening) at weeks 16 and 24, with a mean change of 2.23 (95\% CI 0.93-3.54) from baseline to week 24 (Fig. 5c, online suppl. Table S2).

The J-CGIC score was generally favorable during the study (Fig. $5 \mathrm{~d}$, online suppl. Table S4). At week 24, $40.7 \%$ of enrolled patients (48/118) were judged to have experienced an improvement (slightly improved, improved, or marked improved), while $36.4 \%$ of enrolled patients $(43 / 118)$ were judged to have no change in disease. Only $10.2 \%$ of enrolled patients $(12 / 118)$ were judged to have worsening disease by week 24 . 
Table 3. Adverse events

\begin{tabular}{lccc}
\hline AE (by preferred term) & \multicolumn{3}{l}{ Study period $(n=118)$} \\
\cline { 2 - 3 } & titration & maintenance & overall \\
\hline Patients with any AE & $54(45.8)$ & $63(53.4)$ & $88(74.6)$ \\
Dermatitis contact & $9(7.6)$ & $9(7.6)$ & $18(15.3)$ \\
Nasopharyngitis & $4(3.4)$ & $10(8.5)$ & $12(10.2)$ \\
Application site pruritus & $8(6.8)$ & $2(1.7)$ & $10(8.5)$ \\
Erythema & $5(4.2)$ & $5(4.2)$ & $10(8.5)$ \\
Pruritus & $4(3.4)$ & $6(5.1)$ & $10(8.5)$ \\
Application site erythema & $8(6.8)$ & $2(1.7)$ & $9(7.6)$ \\
Rash & $2(1.7)$ & $4(3.4)$ & $6(5.1)$ \\
Nausea & $2(1.7)$ & $3(2.5)$ & $5(4.2)$ \\
Diarrhea & $3(2.5)$ & $1(0.8)$ & $4(3.4)$ \\
Vomiting & $1(0.8)$ & $2(1.7)$ & $3(2.5)$ \\
Application site rash & $3(2.5)$ & 0 & $3(2.5)$ \\
Dementia & $1(0.8)$ & $2(1.7)$ & $3(2.5)$ \\
Dementia Alzheimer's type & $1(0.8)$ & $2(1.7)$ & $3(2.5)$ \\
Insomnia & $1(0.8)$ & $2(1.7)$ & $3(2.5)$ \\
Hypertension & $2(1.7)$ & $1(0.8)$ & $3(2.5)$ \\
\hline
\end{tabular}

Values indicate number (\%) of patients. AE, adverse event.

Table 4. Serious adverse events

\begin{tabular}{llll}
\hline SAE (by system organ class and preferred term) & \multicolumn{2}{l}{ Study period $(n=118)$} & \\
\cline { 2 - 4 } & titration & maintenance & overall \\
\hline Patients with $\geq 1$ SAE & $1(0.8)$ & $4(3.4)$ & $5(4.2)$ \\
\hline Gastrointestinal disorders & 0 & $1(0.8)$ & $1(0.8)$ \\
$\quad$ Ileus & 0 & $1(0.8)$ & $1(0.8)$ \\
$\quad$ Pancreatitis acute & 0 & $1(0.8)$ & $1(0.8)$ \\
\hline Infections and infestations & 0 & $1(0.8)$ & $1(0.8)$ \\
$\quad$ Herpes zoster & 0 & $1(0.8)$ & $1(0.8)$ \\
$\quad$ Pneumonia & 0 & $1(0.8)$ & $1(0.8)$ \\
\hline Metabolism and nutrition disorders & 0 & $2(1.7)$ & $2(1.7)$ \\
$\quad \begin{array}{lll}\text { Dehydration } \\
\text { Hypoglycemia }\end{array}$ & 0 & $1(0.8)$ & $1(0.8)$ \\
\hline Musculoskeletal and connective tissue disorders & 0 & $1(0.8)$ & $1(0.8)$ \\
$\quad$ Arthralgia & 0 & $1(0.8)$ & $1(0.8)$ \\
\hline Neoplasms benign, malignant, and unspecified & $1(0.8)$ & 0 & $1(0.8)$ \\
(including cysts and polyps) & & $1(0.8)$ & $1(0.8)$ \\
$\quad$ Breast cancer & $1(0.8)$ & 0 & $1(0.8)$ \\
\hline
\end{tabular}

Values indicate number (\%) of patients. SAE, serious adverse event.

\section{Safety}

Overall, 88 patients (74.6\%) experienced at least one AE during the study, with AEs occurring in 54 patients (45.8\%) in the 8-week titration period and in 63 patients $(53.4 \%)$ in the 16-week maintenance period (Table 3). The most common AEs were skin-related AEs. A total of 8 serious AEs occurred in 5 patients (4.2\%), most of which occurred in the mainte- 
Table 5. Adverse events leading to study discontinuation

\begin{tabular}{llcc}
\hline AE (by preferred term) & \multicolumn{2}{l}{ Study period $(n=118)$} & \\
\cline { 2 - 4 } & titration & maintenance & overall \\
\hline Patients with an AE leading to study discontinuation & $7(5.9)$ & $7(5.9)$ & $14(11.9)$ \\
Dementia & 0 & $2(1.7)$ & $2(1.7)$ \\
Dementia Alzheimer's type & $1(0.8)$ & $1(0.8)$ & $2(1.7)$ \\
Dermatitis contact & $1(0.8)$ & $1(0.8)$ & $2(1.7)$ \\
Dermatitis allergic & $1(0.8)$ & $1(0.8)$ & $2(1.7)$ \\
Breast cancer (SAE) & $1(0.8)$ & 0 & $1(0.8)$ \\
Application site erythema & $1(0.8)$ & 0 & $1(0.8)$ \\
Rash & $1(0.8)$ & 0 & $1(0.8)$ \\
Compression fracture & $1(0.8)$ & 0 & $1(0.8)$ \\
Pneumonia (SAE) & 0 & $1(0.8)$ & $1(0.8)$ \\
Neuropsychiatric symptoms & 0 & $1(0.8)$ & $1(0.8)$ \\
\hline
\end{tabular}

Values indicate number (\%) of patients. AE, adverse event; SAE, serious adverse event.

Table 6. Adverse events of special interest

\begin{tabular}{llll}
\hline \multirow{2}{*}{ AE (by preferred term) } & \multicolumn{2}{l}{ Study period $(n=118)$} & \\
\cline { 2 - 4 } & titration & maintenance & overall \\
\hline Total & $29(24.6)$ & $16(13.6)$ & $40(33.9)$ \\
\hline Application site skin reactions and irritations & $26(22.0)$ & $12(10.2)$ & $36(30.5)$ \\
$\quad$ Dermatitis contact & $9(7.6)$ & $9(7.6)$ & $18(15.3)$ \\
Application site pruritus & $8(6.8)$ & $2(1.7)$ & $10(8.5)$ \\
Application site erythema & $8(6.8)$ & $2(1.7)$ & $9(7.6)$ \\
Application site rash & $3(2.5)$ & 0 & $3(2.5)$ \\
Dermatitis allergic & $1(0.8)$ & $1(0.8)$ & $2(1.7)$ \\
Application site dermatitis & $1(0.8)$ & 0 & $1(0.8)$ \\
Application site eczema & $1(0.8)$ & 0 & $1(0.8)$ \\
\hline GI symptoms & $4(3.4)$ & $5(4.2)$ & $9(7.6)$ \\
Nausea & $2(1.7)$ & $3(2.5)$ & $5(4.2)$ \\
Diarrhea & $3(2.5)$ & $1(0.8)$ & $4(3.4)$ \\
Vomiting & $1(0.8)$ & $2(1.7)$ & $3(2.5)$ \\
\hline
\end{tabular}

Values indicate number (\%) of patients. AE, adverse event; GI, gastrointestinal.

nance period (Table 4). Fourteen patients discontinued due to AEs (Table 5) and 12 patients required a dose reduction due to an AE (online suppl. Table S5). Dementia-related and skinrelated AEs were the most common types of AEs resulting in discontinuation of treatment or a dose reduction.

The incidences of skin-related and GI-related AEs were examined as AEs of special interest (Table 6). AEs of special interest occurred in a total of 40 patients (33.9\%) and included application site skin reactions or irritations in 36 patients (30.5\%) and GI symptoms in 9 patients (7.6\%). The incidence of skin-related AEs was numerically higher in the titration period $(22.0 \%)$ than in the maintenance period (10.2\%) The incidence of skin-related AEs was similar between patients who used a moisturizer at baseline and those who did not (16/49 [32.7\%] vs. 20/69 [29.0\%]) (Table 7). 
Ueda et al.: Switching to Rivastigmine Transdermal Patch in Mild to Moderate Alzheimer's Disease

Table 7. Incidence of application site skin-related adverse events according to use of moisturizer at baseline (week 1-24)

\begin{tabular}{|c|c|c|}
\hline \multirow[t]{2}{*}{ AE (by preferred term) } & \multicolumn{2}{|c|}{$\begin{array}{l}\text { Moisturizer use at baseline }(n= \\
118)\end{array}$} \\
\hline & yes $(n=49)$ & no $(n=69)$ \\
\hline Total & $16(32.7)$ & $20(29.0)$ \\
\hline Dermatitis contact & $6(12.2)$ & $12(17.4)$ \\
\hline Application site pruritus & $6(12.2)$ & $4(5.8)$ \\
\hline Application site erythema & $7(14.3)$ & $2(2.9)$ \\
\hline Application site rash & 0 & $3(4.3)$ \\
\hline Dermatitis allergic & $2(4.1)$ & 0 \\
\hline Application site dermatitis & $1(2.0)$ & 0 \\
\hline Application site eczema & 0 & $1(1.4)$ \\
\hline
\end{tabular}

Values indicate number (\%) of patients. AE, adverse event.

Table 8. Caregiver assessment of usability $(n=118)$

\begin{tabular}{lc} 
For caregivers responding that the patch was "very easy to use" or "easy to use"a & \\
Easier to follow medication schedule & $50(42.4)$ \\
Can provide visual reassurance that the medication is being taken & $34(28.8)$ \\
Can help minimize caregiver's burden for preparing and administering & \\
$\quad$ medication & $23(19.5)$ \\
None of the above applies & $1(0.8)$ \\
\hline For caregivers responding that the patch was "not easy to use" or "not easy to use at all”a & \\
Oral drugs can help minimize the caregiver's burden for preparing and & \\
$\quad$ administering medications & $26(22.0)$ \\
Easier to check treatment compliance of oral medication & $8(6.8)$ \\
Easier to follow medication schedule for oral medications & $5(4.2)$ \\
None of the above applies & $8(6.8)$
\end{tabular}

Values indicate number (\%) of patients. ${ }^{\text {a }}$ Multiple responses were allowed.

In terms of GI-related AEs, there was no apparent difference in the incidence of GI symptoms between titration and maintenance periods (3.4 vs. 4.2\%) (Table 6). However, the incidence of GI symptoms was slightly greater in patients in the lower-weight bands than in patients in the higher-weight bands (online suppl. Table S6), perhaps reflecting greater systemic exposure in lower-weight patients.

\section{Usability/Preference}

More than half of the caregivers reported that rivastigmine transdermal patch was very easy $(32.5 \%)$ or easy $(22.2 \%)$ to use compared with oral tablets, while $10.3 \%$ reported no change (Fig. 6). The other one-third of caregivers thought that rivastigmine transdermal patch was not easy to use (30.8\%) or not easy to use at all (4.3\%). The most common reason cited by the caregivers for the ease of use was that rivastigmine transdermal patch is easier to follow the medication schedule $(42.4 \%)$ and that it provides visual reassurance that the medication is being taken (28.8\%) (Table 8). The most common reason given by caregivers to explain why rivastigmine transdermal patch was not easy to use was that oral drugs can reduce their burden for preparing and administering medication. 
Ueda et al.: Switching to Rivastigmine Transdermal Patch in Mild to Moderate Alzheimer's Disease

Fig. 6. Caregiver preference for rivastigmine transdermal.

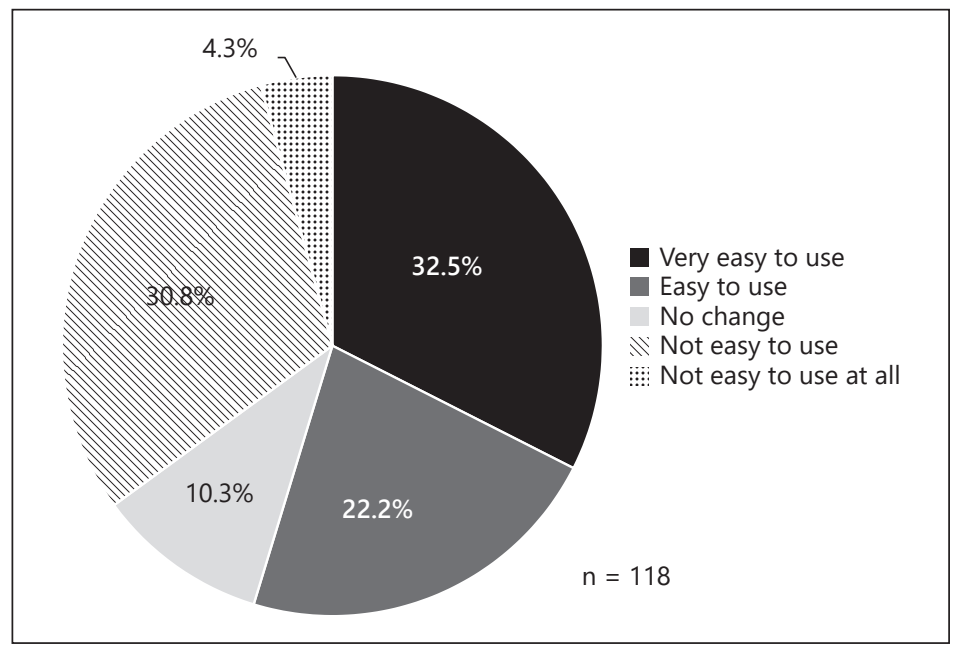

\section{Discussion}

\section{Efficacy}

In this study, we examined the efficacy and safety of a one-step switch from an oral ChEI to rivastigmine transdermal patch in patients with mild to moderate AD in hospitals and clinics in Japan. The efficacy was assessed using a battery of clinical tests, namely MMSE, NPI-10, QOL-AD, modified Crichton Scale, and J-CGIC. The primary endpoint, MMSE total score, showed a decrease of 0.36 from baseline through to week 24 that is not significantly different from the baseline, suggesting that cognitive function was maintained after switching to rivastigmine transdermal patch. There was a small improvement at week 8 , which might represent a short-term effect of medication switch, although we cannot exclude the possibility of a learning effect. When we used the mixed-effects model to compare the changes in MMSE between the 24-week period before baseline and the 24-week study period, we observed that a marked MMSE deterioration in the pre-study period was halted and stabilized after switching to rivastigmine transdermal patch. Because 112/118 patients (94.9\%) were enrolled due to a lack or loss of efficacy with oral ChEIs, these results suggest the clinical usefulness of switching to rivastigmine transdermal patch in patients who do not show a good response to oral ChEIs.

It is intriguing to note that the response to rivastigmine tended to be greater in patients with relatively mild AD, such as those within high baseline MMSE total scores. This finding suggests that greater efficacy could be achieved by switching from oral ChEIs to rivastigmine transdermal patch at an earlier stage. There was a tendency towards a greater responsiveness among the patients enrolled due to an MMSE score decline, compared to those enrolled due to worsening in BPSD or ADL. This may be attributable to the use of MMSE rather than NPI-10 or modified Crichton Scale for evaluating the responsiveness to ChEIs. Nevertheless, these results suggest the importance of periodic follow-up of patients using MMSE or other cognitive batteries as part of their medical examination, along with the evaluation of ADL and BPSD as a basis for deciding on whether/when to switch from an oral ChEI to rivastigmine transdermal patch.

In terms of other efficacy endpoints, we observed an improvement in the NPI total score within about 8 weeks of starting treatment, which returned to baseline level by the end of the study. However, discordant with the other efficacy outcomes, there was a slight worsening in the modified Crichton Scale during the course of the study, which might represent the accu- 
Ueda et al.: Switching to Rivastigmine Transdermal Patch in Mild to Moderate Alzheimer's Disease

mulated burden of long-term care. Indeed, it has been reported that the caregiver's evaluation of the patient's ADL worsens as the level or the period of burden increases [12]. The greater expectation for a positive treatment outcome by caregivers as compared with doctors [13] might also contribute to the discordant evaluations in the present study. It has been reported that caregivers who are unrelated to their patient perceive their patient's abilities to be higher than do caregivers who are closer or related to patients, such as spouses [14]. This may also be related to differences in burden or the level of expectation. These findings stress the importance of providing education and support to caregivers. Indeed, it has been reported that caregiver psychoeducational intervention can increase their own QOL and reduce their burden $[15,16]$.

QOL assessed by the caregivers and patients was essentially maintained over time. This may reflect stability of QOL, or alternatively indicate that QOL-AD may not be sensitive to short-term changes. The lower rating of QOL-AD scores reported by caregivers versus the patients' own responses agrees with the worsening of the modified Crichton Scale, which may be related to the patient's lack of awareness of their symptoms and/or the burden imposed on caregivers.

A notable finding, however, relates to the discordant response rates for J-CGIC and MMSE, as about $40 \%$ of patients experienced no change/improvement in MMSE compared with $\sim 90 \%$ for J-CGIC. One explanation is that MMSE reflects part of the overall dementia symptoms, and that clinicians might perceive an overall improvement if BPSD and ADL are maintained, even in patients with worsening cognitive function.

\section{Safety}

In the present study, we did not observe any unanticipated AEs that were reported in previous studies. This suggests that switching from an oral ChEI to rivastigmine transdermal patch can be safely performed using a one-step titration method. The safety profile in this study was consistent with that reported in a prior clinical trial in Japan comparing the efficacy and safety of one-step and three-step titration algorithms [10] and another study that used a three-step titration algorithm with the final doses of $18 \mathrm{mg}\left(9.5 \mathrm{mg} / 24 \mathrm{~h}, 10 \mathrm{~cm}^{2}\right)$ and $9 \mathrm{mg}$ (4.6 mg/24 h, $5 \mathrm{~cm}^{2}$ ) [10].

As expected from the results of prior studies in Japan [10,11], skin-related AEs were the most common AEs in this study. However, the incidences of these AEs were lower in the present study than in the prior studies. For example, application site erythema and application site pruritus occurred in 7.6 and $8.5 \%$ of patients, respectively, in the present study versus 39.4 and $34.8 \%$ in the first 24 -week study in Japan at the same dose of rivastigmine [10]. This might suggest improvements in managing skin symptoms or preventative therapies, such as early application of moisturizers. Indeed, in the present study, the incidence of skin-related AEs was similar between inpatients who used moisturizers at baseline and those who did not. This could be explained by an improvement in skin care if moisturizers were preferentially applied to patients with sensitive skin who might be prone to skin-related AEs. It is also possible that some patients did not choose to or could not use moisturizers, despite experiencing skin symptoms. Considering that the incidence of skin-related AEs was similar between the titration and maintenance phase, it seems clear to us that clinicians and caregivers should be vigilant for and consider preventative treatments during titration and ongoing therapy with rivastigmine transdermal patch.

The other AEs of special interest were related to GI symptoms. Due to the study design in which patients switched directly from their previous oral ChEI to $9 \mathrm{mg}$ rivastigmine transdermal patch, patients who experienced GI AEs with their prior therapy were excluded from the study. Despite this exclusion, approximately $8 \%$ of subjects experienced GI AEs. This 
Ueda et al.: Switching to Rivastigmine Transdermal Patch in Mild to Moderate Alzheimer's Disease

suggests that the three ChEIs do not cause GI AEs in the same way, and that it is important to consider the benefits and risks when switching between ChEIs.

\section{Compliance}

The overall compliance rate was high, with an overall mean compliance rate of $97.6 \%$, and the majority of patients had compliance of $75-100 \%$. This high compliance rate may reflect the administration of rivastigmine transdermal patch by a caregiver, or it may be because ongoing and regular visits with clinicians in the clinical trial setting and better understanding of the disease and treatment by caregivers may encourage adherence and compliance to the prescribed treatment.

\section{Usability}

In terms of usability, $54.2 \%$ of the caregivers reported that rivastigmine transdermal patch was easy or very easy to use. This compares with a value of $61 \%$ in a prior study in Japan [10]. The slightly lower proportion in the present study might be because very few patients were enrolled in the study due to difficulty with continuing their previous oral medication; patients experiencing difficulty in taking an oral medication might experience a greater benefit of switching to a transdermal patch.

\section{Comparison with Previous Studies of Switching among ChEIs}

Based on comprehensive evaluations such as network meta-analysis and systematic review, it is thought that rivastigmine, donepezil, and galantamine have similar effects on cognitive function $[17,18]$. However, the responses of each patient to these ChEIs might vary due to differences in the mechanism of action, metabolism, pharmacokinetics, and the treatment compliance of these ChEIs that may be affected by the formulation type and the frequency of administration. Thus, patients who are unresponsive to one agent may respond to a different $\mathrm{ChEI}$ [4]. In fact, in a prospective study in which patients with a poor response to an oral ChEI were switched to rivastigmine transdermal patch for 6 months, the MMSE score was stable or improved in over half of the patients [8]. In addition, a post hoc analysis of the EVOLUTION study revealed improvements in BPSD, especially in delusions and anxiety items, in moderate AD patients after switching from an oral ChEI to rivastigmine transdermal patch [19]. The results of these studies are consistent with the findings of the current study.

The benefit of switching ChEIs is not limited to cases where oral ChEIs are switched to rivastigmine transdermal patch. For example, a post-marketing phase IV study revealed an improvement in cognitive function when patients were switched from donepezil to galantamine [20]. Although our study is limited to a one-directional switch from donepezil or galantamine to rivastigmine, considering the potential difference in the individual patient's response to each ChEI, we believe that switching to other drugs should be considered for patients who do not respond to rivastigmine. Many of the switching studies conducted to date involved switching from oral ChEIs to rivastigmine transdermal patch. To demonstrate the usefulness of switching among ChEIs in general, it is necessary to conduct a study in which patients switch among the three ChEIs under the same conditions.

\section{Limitations}

A main limitation of this study is that we did not include a control group (e.g., continuation of prior oral $\mathrm{ChEI}$, which may limit the validity of the analyses. In addition to the pharmacological action of rivastigmine, the expectations of patients and caregivers for the new medication and environmental changes associated with switching in the clinical trial setting, 
Ueda et al:: Switching to Rivastigmine Transdermal Patch in Mild to Moderate Alzheimer's Disease

such as social and psychological influences of the trial environment on patients and caregivers, might have affected the results of this study.

\section{Conclusions}

In conclusion, within-class switching from an oral ChEI to rivastigmine transdermal patch might be an efficacious and tolerable option for AD patients showing a poor or limited response to a prior oral ChEI.

\section{Acknowledgement}

The authors thank the following principal investigators and their staff for conducting the study: Yasuhiro Kawase (Kawase Neurology Clinic), Eiji Kirino (Juntendo University), Yuri Kitamura (Nanohana Clinic), Tomoyuki Ono (Takesato Hospital), Tsuneyoshi Ota (Juntendo University), Takahisa Shimano (Juntendo University), Masashi Takita (Takita Memory Mental Clinic), Yoshio Tsuboi (Fukuoka University). The authors also thank Nicholas D. Smith and EMC K.K. for medical writing support, Asahi C\&I for supporting the generation of figures and tables, and Haruhisa Okawa, a full-time employee of Novartis K.K., for medical writing support and the management of manuscript preparation.

\section{Statement of Ethics}

The study protocol was reviewed by the institutional review board at each participating center, and the study was conducted in accordance with ICH Harmonized Tripartite Guidelines for Good Clinical Practice with applicable regulations in Japan and the Declaration of Helsinki. All patients, or their caregiver, provided written informed consent before enrolment. The study was registered on ClinicalTrials.gov (NCT02703636).

\section{Disclosure Statement}

This study was funded by Novartis Pharma K.K. Kengo Ueda, Kazuki Kiyose, and Kazuhiro Toriyama are full-time employees of Novartis Pharma K.K. Ankita Shekhawat and Ayan Das Gupta are full-time employees of Novartis Healthcare Pvt. Ltd. In the pastyear, Sadao Katayama has received speaking fees from Novartis Pharma K.K., and research fees including payment for clinical trials from Eli Lilly Japan K.K., Biogen Japan Ltd., Chugai Pharmaceutical Co., Ltd., Eisai Co., Ltd., and Boehringer Ingelheim Japan. Tetsuaki Arai has received speaking fees from Eisai Co., Ltd. and Daiichi Sankyo Co., Ltd. Nobuo Furuta has received speaking fees from Janssen Pharmaceutical K.K. Makio Takahashi has received lecture fees from Eisai Co., Ltd., Ono Pharmaceutical Co., Ltd., Janssen Pharmaceutical K.K. and Novartis Pharma K.K. Toshihisa Tanaka has received fees for research from Biogen Japan Ltd., MSD K.K., Shionogi \& Co., Ltd., and speaking fees from Ono Pharmaceutical Co., Ltd., Eisai Co., Ltd., and Meiji Seika Pharma Co., Ltd. Yu Nakamura has received speaking fees from Daiichi Sankyo Co., Ltd., Ono Pharmaceutical Co., Ltd., Takeda Pharmaceutical Co., Ltd., Eisai Co., Ltd., Novartis Pharma K.K., Eli Lilly Japan K.K., MSD K.K., and research fees including payment for clinical trials from Daiichi Sankyo Co., Ltd., and Biogen Japan Ltd. The other authors have no conflict of interest to declare. 


\title{
Funding Sources
}

\author{
This study was fully funded by Novartis Pharma K.K.
}

\section{Author Contributions}

Kengo Ueda, Kazuki Kiyose, and Kazuhiro Toriyama contributed to the conception of the work and the interpretation of data. Ankita Shekhawat and Ayan Das Gupta contributed to the analysis of data for this work. Yu Nakamura contributed to the conception of the work, and the acquisition and interpretation of data. The other authors contributed to the acquisition and interpretation of data. All authors contributed to the drafting of the work and its critical revision.

\section{References}

1 Takeda M, Tanaka T, Okochi M. New drugs for Alzheimer's disease in Japan. Psychiatry Clin Neurosci. 2011 Aug; 65(5):399-404.

2 Gauthier S, Emre M, Farlow MR, Bullock R, Grossberg GT, Potkin SG. Strategies for continued successful treatment of Alzheimer's disease: switching cholinesterase inhibitors. Curr Med Res Opin. 2003;19(8):707-14.

3 Spalletta G, Caltagirone C, Padovani A, Sorbi S, Attar M, Colombo D, et al.; E V O L U T I O N study Working Group. Cognitive and affective changes in mild to moderate Alzheimer's disease patients undergoing switch of cholinesterase inhibitors: a 6-month observational study. PLoS One. 2014 Feb;9(2):e89216.

4 Blesa R, Toriyama K, Ueda K, Knox S, Grossberg G. Strategies for continued successful treatment in patients with Alzheimer's disease: an overview of switching between pharmacological agents. Curr Alzheimer Res. 2018; 15(10):964-74.

5 Massoud F, Desmarais JE, Gauthier S. Switching cholinesterase inhibitors in older adults with dementia. Int Psychogeriatr. 2011 Apr;23(3):372-8.

6 Sadowsky CH, Dengiz A, Olin JT, Koumaras B, Meng X, Brannan S; US38 study group. Switching from donepezil tablets to rivastigmine transdermal patch in Alzheimer's disease. Am J Alzheimers Dis Other Demen. 2009 Jun-Jul;24(3):267-75.

7 Sadowsky CH, Farlow MR, Meng X, Olin JT. Safety and tolerability of rivastigmine transdermal patch compared with rivastigmine capsules in patients switched from donepezil: data from three clinical trials. Int J Clin Pract. 2010 Jan;64(2):188-93.

8 Cagnin A, Cester A, Costa B, Ermani M, Gabelli C, Gambina G; SWITCH study working group. Effectiveness of switching to the rivastigmine transdermal patch from oral cholinesterase inhibitors: a naturalistic prospective study in Alzheimer's disease. Neurol Sci. 2015 Mar;36(3):457-63.

9 Han HJ, Lee JJ, Park SA, Park HY, Kim JE, Shim YS, et al. Efficacy and safety of switching from oral cholinesterase inhibitors to the rivastigmine transdermal patch in patients with probable Alzheimer's disease. J Clin Neurol. 2011 Sep;7(3):137-42.

10 Nakamura Y, Imai Y, Shigeta M, Graf A, Shirahase T, Kim H, et al. A 24-week, randomized, double-blind, placebocontrolled study to evaluate the efficacy, safety and tolerability of the rivastigmine patch in Japanese patients with Alzheimer's disease. Dement Geriatr Cogn Disord Extra. 2011 Jan;1(1):163-79.

11 Nakamura Y, Strohmaier C, Tamura K, Kataoka N, Nakano M, Oda S, et al. A 24-week, randomized, controlled study to evaluate the tolerability, safety and efficacy of 2 different titration schemes of the rivastigmine patch in Japanese patients with mild to moderate Alzheimer's disease. Dement Geriatr Cogn Disord Extra. 2015 Sep;5(3): 361-74.

12 Zanetti 0, Geroldi C, Frisoni GB, Bianchetti A, Trabucchi M. Contrasting results between caregiver's report and direct assessment of activities of daily living in patients affected by mild and very mild dementia: the contribution of the caregiver's personal characteristics. J Am Geriatr Soc. 1999 Feb;47(2):196-202.

13 Rockwood K, Graham JE, Fay S; ACADIE Investigators. Goal setting and attainment in Alzheimer's disease patients treated with donepezil. J Neurol Neurosurg Psychiatry. 2002 Nov;73(5):500-7.

14 Cacchione PZ, Powlishta KK, Grant EA, Buckles VD, Morris JC. Accuracy of collateral source reports in very mild to mild dementia of the Alzheimer type. J Am Geriatr Soc. 2003 Jun;51(6):819-23.

15 Terayama H, Sakurai H, Namioka N, Jaime R, Otakeguchi K, Fukasawa R, et al. Caregivers' education decreases depression symptoms and burden in caregivers of patients with dementia. Psychogeriatrics. 2018 Sep; 18(5): 327-33.

16 Piersol CV, Canton K, Connor SE, Giller I, Lipman S, Sager S. Effectiveness of interventions for caregivers of people with Alzheimer's disease and related major neurocognitive disorders: a systematic review. Am J Occup Ther 2017;71(5):7105180020p1-7105180020p10. 
17 Hansen RA, Gartlehner G, Webb AP, Morgan LC, Moore CG, Jonas DE. Efficacy and safety of donepezil, galantamine, and rivastigmine for the treatment of Alzheimer's disease: a systematic review and meta-analysis. Clin Interv Aging. 2008;3(2):211-25.

18 Kobayashi H, Ohnishi T, Nakagawa R, Yoshizawa K. The comparative efficacy and safety of cholinesterase inhibitors in patients with mild-to-moderate Alzheimer's disease: a Bayesian network meta-analysis. Int J Geriatr Psychiatry. 2016 Aug;31(8):892-904.

19 Colombo D, Caltagirone C, Padovani A, Sorbi S, Spalletta G, Simoni L, et al. Gender differences in neuropsychiatric symptoms in mild to moderate Alzheimer's disease patients undergoing switch of cholinesterase inhibitors: a post hoc analysis of the EVOLUTION Study. J Womens Health (Larchmt). 2018 Nov;27(11):1368-77.

20 Takahashi N, Takahashi M, Shu T, Izumoto T, Saito T. Efficacy and safety of galantamine hydrobromide in patients with dementia due to Alzheimer's disease who responded poorly to treatment with donepezil: results of a postmarketing clinical trial [In Japanese]. Jpn J Geriatr Psychiatry. 2014;25(1):70-86. 